\title{
Appropriate Use and Outcomes of In-Hospital Telemetry: Implications Regarding the Contemporary Code Blue
}

\author{
Ronald F Espinosa, DO, Christopher A Woolley II, MD, Scot W Nolan, DNP, RN, Jill M \\ Waalen, MD, MPH and Bruce J Kimura, MD, FACC ${ }^{*}$
}

Department of Cardiology, Graduate Medical Education and Critical Care, Scripps Mercy Hospital, USA

*Corresponding author: Bruce J Kimura, MD, FACC, Medical Director, Cardiovascular Imaging Lab, Scripps Mercy Hospital; Clinical Professor (non-salaried), University of California, 4077 Fifth Avenue, MER74, San Diego, California 92103, USA

\begin{abstract}
Background: Although practice standards for telemetry reflect the historical benefit of early monitoring for arrhythmias in select admissions, few data exist on the initial appropriateness of telemetry for contemporary inpatients who then later suffered a "code blue." As telemetry monitoring is a significant resource, we sought to review appropriate use, detection of causative arrhythmias, and the timing and outcomes of telemetry use in code blues.

Methods: Over a 3-year period in a 300-bed teaching hospital, data that had been recorded from all inpatient ward "code blues" were reviewed and initial rhythm categorized as shockable or non-shockable. The presence and length of telemetry prior to the code, initial appropriateness of telemetry by American Heart Association practice standards, and presence of a causative arrhythmia were recorded. Associations of 22 clinical variables with return of spontaneous circulation and survival to hospital discharge were assessed in bivariate and multivariable models.

Results: Of 177 code blues, $91 \%$ were on telemetry of which $94 \%$ were guideline appropriate. Telemetry vs. nonmonitored patients were older $(70 \mathrm{y} \pm 15.7$ vs. $59 \mathrm{y} \pm 15.5$; $p=0.01$ ) and demonstrated no significant difference in return of spontaneous circulation nor survival-to-discharge. Non-shockable rhythms were responsible for the majority of code blue events $(86 \%)$. The best multivariable model for survival-to-discharge included younger age, lack of indication for telemetry, lower admission troponin levels, and a shorter length of telemetry prior to the code.

Conclusions: In our hospital, most patients who suffered code blue events had been appropriately placed on telemetry but demonstrated non-shockable etiologies.
\end{abstract}

\section{Keywords}

Telemetry, Electrocardiographic, Cardiac arrest, In-hospital arrest, Code-blue, Advanced cardiac life support

\section{Introduction}

In 2017, an update to the 2014 American Heart Association (AHA) statement entitled "Practice Standards for Electrocardiographic Monitoring in Hospital Settings" re-emphasized the value of telemetry as an early warning system to protect patients from cardiac arrest and malignant arrhythmias [1]. Although a strong evidence-basis is lacking, the current guidelines demonstrate high sensitivity in identifying the patient at increased risk for life-threatening arrhythmias $[2,3]$. Subsequent studies that examined the association between appropriate telemetry use and survivalto-discharge have revealed conflicting data $[4,5]$. As the etiology of the in-hospital code blues may have evolved over the years and have implications for the cost-effective use of telemetry, we sought to review appropriate use of telemetry, detection of causative arrhythmias, and the timing and outcomes for code blues in a tertiary hospital $[6,7]$.

\section{Methods}

The study was a retrospective cohort analysis approved by the Scripps Institutional Review Board (Scripps Health, San Diego, \#17-6989). Data was collected for consecutive patients $>18$ years of age who underwent a code blue resuscitation attempt during 2013-2016 at Scripps Mercy Hospital, a 300-bed tertiary care, teaching community hospital. A code blue resuscitation attempt was defined as the activation of the hospital's code blue team and application of

Citation: Espinosa RF, Christopher AWII, Nolan SW, Waalen JM, Kimura BJ (2021) Appropriate Use and Outcomes of In-Hospital Telemetry: Implications Regarding the Contemporary Code Blue. Int J Clin Cardiol 8:235. doi.org/10.23937/2378-2951/1410235

Accepted: September 01, 2021: Published: September 03, 2021

Copyright: (C) 2021 Espinosa RF, et al. This is an open-access article distributed under the terms of the Creative Commons Attribution License, which permits unrestricted use, distribution, and reproduction in any medium, provided the original author and source are credited. 
advanced cardiac life support to an unresponsive patient. Intensive care unit, operating room and emergency room code blue activations were excluded. Patient telemetry, as applied throughout the hospital, was continuously monitored using (Welch Allyn Micropaq Model $40 \times$ ) by 3 dedicated telemetry technicians from a central unit. Fifty-three percent $(160 / 300)$ of noncritical beds were capable of telemetry monitoring at our institution. Telemetry technicians alerted nursing staff of the presence of any arrhythmia after manual review of the rhythm but typically did not activate the code blue team. The decision for code blue activation was then based on the patient's immediate clinical status in conjunction with telemetry monitoring data. The code blue resuscitation team consisted of either an emergency room or intensive care physician, respiratory therapist, and nursing trained in critical care and utilized standard advanced cardiac life support (ACLS) protocols. Data was recorded on standardized codeblue flow sheets by a designated critical care trained nurse and were later reviewed for this study. Code blue activations were categorized into either 1) Shockable, defined as the initial rhythm of ventricular tachycardia, ventricular fibrillation, and torsades de pointes or 2) Non-shockable, defined as sinus tachycardia, pulseless electrical activity, atrial fibrillation or flutter with rapid ventricular response, bradycardia/asystole, or seizures. In the analysis, shockable causes were scrutinized for temporal and/or clinical evidence of being the primary cause of the patient to be unresponsive or rapidly deteriorate. Appropriate telemetry implementation was assessed based on the 2014 AHA guidelines and recorded as Class I (indicated as patient has a significant risk of arrhythmia), Class II (may be of benefit in some patient but is not considered essential for all patients), or Class III (not indicated as a patient's risk of a serious event is so low that monitoring has no therapeutic benefit) [1]. Either admission or earliest pre-cardiac arrest laboratory, historical, or study variables and post-cardiac arrest outcome variables were obtained through electronic chart review, including survival-todischarge (excluding those discharged to hospice or comfort care), return of spontaneous circulation, days on telemetry prior to the code, and multiple pre-arrest lab or clinical values including troponin, creatine kinase, creatinine, hemoglobin, potassium, blood urea nitrogen, hemoglobin, calcium, magnesium, heart rate, corrected QT interval, initial heart rhythm, white blood cell count, systolic blood pressure, diastolic blood pressure. Coronary artery disease, atrial fibrillation, congestive heart failure (left ventricular ejection fraction < 55\%), chronic kidney disease, and diabetes mellitus were recorded as existing co-morbid medical conditions if they were stated on admission as established diagnosis, either through history taking or chart review.

\section{Statistical Analysis}

Categorical variables are presented as percentages and were compared between groups using the Fisher's exact test. Continuous variables are expressed as mean and standard deviations and were compared between groups using the two-sided Student t-test. In order to investigate clinical variables independently associated with survival-to-discharge, multivariable logistic regression analysis including presence and length of telemetry, and patient clinical and laboratory variables in SAS 9.2 (Cary, North Carolina). A p-value of $<0.05$ was considered statistically significant.

\section{Figure 1: AHA Categorization}

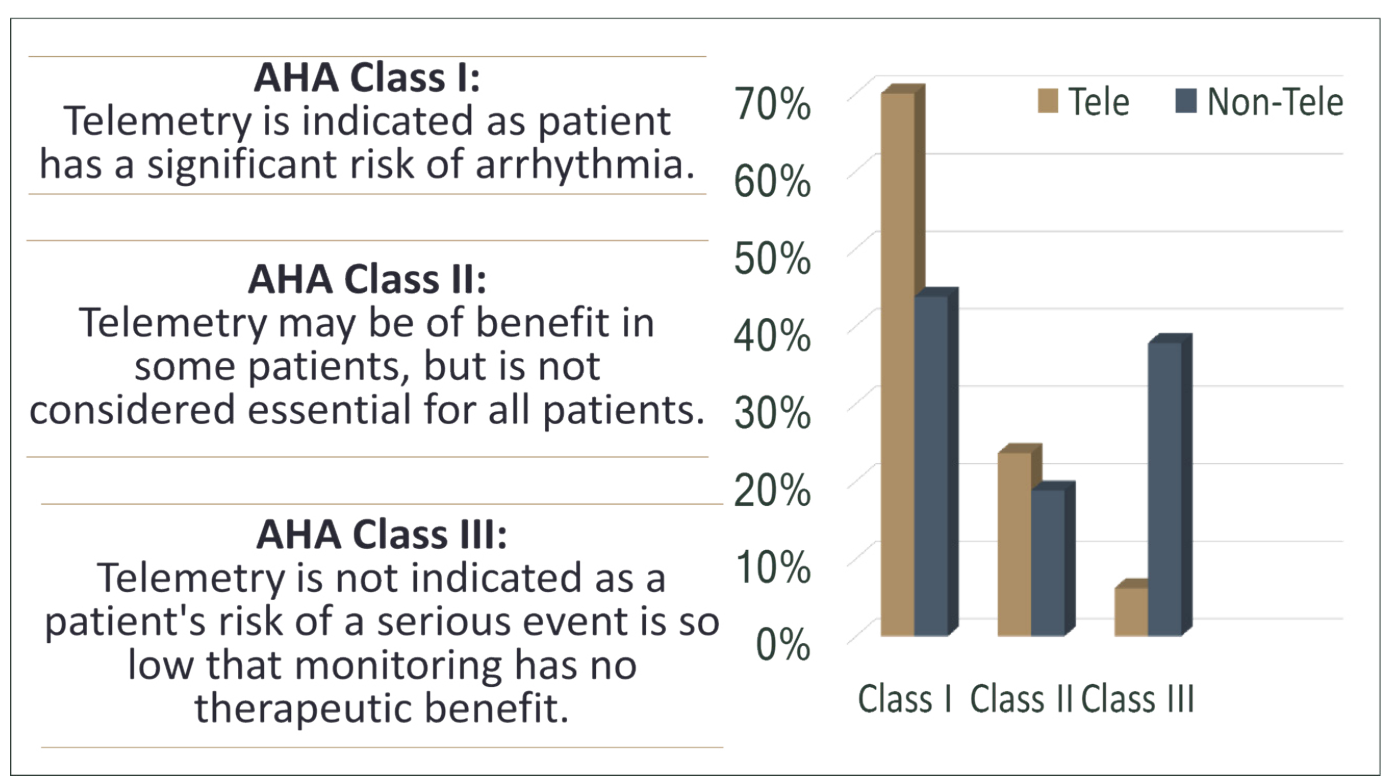

Figure 1: American Heart Association Guideline Classification of Patients in Code Blues.

Bar graph shows the percentage (y-axis) of patients in AHA Class I, II, and III in the group on telemetry (blue) and nonmonitored (orange) $x$-axis. Class I and II indicate appropriate implementation. 


\section{Results}

Of the 177 code blue activations, 161 (91\%) were monitored on telemetry. The mean ( \pm SD) patient age was $65 \pm 16$ years. Telemetry patients were older (70 years \pm 15 vs. 60 years $\pm 15 ; p<0.05)$. Of the code blue events, $94 \%(151 / 161)$ of patients on telemetry either met AHA class I or class II indications for telemetry versus $63 \%(10 / 16)$ of non-telemetry patients (Figure 1 ).

The majority of patients, $86 \%$ (139/161), experienced non-shockable code blue activations. Shockable codes were the minority of code blue activations, occurring in only $14 \%(22 / 161)$ of telemetry monitored patients. In patients with non-shockable code blues, return of spontaneous circulation occurred in $82 \%(114 / 139)$ of telemetry monitored patients versus $88 \%(14 / 16)$, ( $p=$ 0.58 ) of non-monitored patients. Survival to discharge occurred in $35 \%(49 / 139)$ of monitored patients versus $56 \%(9 / 16),(p=0.17)$ of non-monitored patients (Table 1). A statistically significant difference was found in those with non-shockable rhythms, occurring later in the hospital course in patients monitored on telemetry averaging $8.55(\mathrm{SD} \pm 9.3)$ vs. $2.7(\mathrm{SD} \pm 1.9)$ in patients who were not monitored (Table 2). Aside from the presence of coronary artery disease $(41.6 \%$ vs. $12.5 \%$, $p=0.03)$ and age $(69.9 \pm 15.7$ vs. $59.1 \pm 15.5, p=0.01)$, no other statistically significant differences were seen between the two groups (Table 3). Therefore, we found no difference in either return of spontaneous circulation or survival to discharge, in patients with non-shockable causes of code blue activations, despite appropriate telemetry monitoring (Table 1 and Figure 2).

Characteristics significantly associated with survivalto-discharge in univariable models included younger age, lack of AHA indication for telemetry, shorter length of telemetry monitoring prior to the code blue resuscitation event, and a troponin level on admission of $<0.04$ (median value). In a multivariable model including all 4 variables, lack of AHA indication was no longer significantly associated with survival (Table 4).

\section{Discussion}

The current study shows that although the implementation of telemetry detected the majority of patients who experienced a code blue activation, $86 \%$ arrested due to non-shockable causes. The minimal number of patients that suffered a code blue event

Table 1: Code Category, telemetry monitoring status and outcomes of survival to discharge and return of spontaneous circulation.

\begin{tabular}{|c|c|c|c|c|c|c|}
\hline Cause of Code & Telemetry+ & ROSC & STD & Telemetry- & ROSC & STD \\
\hline Total & $161(91 \%)$ & $131(81 \%)$ & $58(36 \%)$ & $16(9 \%)$ & $14(88 \%)$ & $9(56 \%)$ \\
\hline Shockable Rhythms & $22(14 \%)$ & $17(77 \%)$ & $9(41 \%)$ & 0 & 0 & 0 \\
\hline Ventricular tachycardia & 9 & 6 & 5 & 0 & 0 & 0 \\
\hline Ventricular fibrillation & 11 & 9 & 3 & 0 & 0 & 0 \\
\hline Torsades de pointes & 2 & 2 & 1 & 0 & 0 & 0 \\
\hline Non-shockable Rhythms & $139(86 \%)$ & $114(81 \%)$ & $49(35 \%)$ & $16(100 \%)$ & $14(88 \%)$ & $9(56 \%)$ \\
\hline Asystole & 13 & 8 & 3 & 4 & 3 & 0 \\
\hline Pulseless Electrical Activity & 55 & 44 & 12 & 1 & 1 & 1 \\
\hline Atrial Fibrillation/Flutter & 2 & 2 & 2 & 0 & 0 & 0 \\
\hline $\begin{array}{l}\text { Sinus Tachycardia/ } \\
\text { Respiratory Failure }\end{array}$ & 44 & 38 & 16 & 4 & 3 & 3 \\
\hline Bradycardia & 14 & 12 & 9 & 3 & 3 & 1 \\
\hline Opiate Overdose & 3 & 3 & 3 & 3 & 3 & 3 \\
\hline Syncope & 2 & 2 & 2 & 0 & 0 & 0 \\
\hline Seizure & 2 & 2 & 1 & 1 & 1 & 1 \\
\hline Sinus Rhythm & 4 & 3 & 1 & 0 & 0 & 0 \\
\hline
\end{tabular}

CCM: Continuous Cardiac Monitoring; ROSC: Return of Spontaneous Circulation; STD: Survival to Discharge

Table 2: Timing of Code Blue Events.

\begin{tabular}{|l|l|l|l|}
\hline & Telemetry+ $(\mathbf{n = 1 6 1 )}$ & $\begin{array}{l}\text { Telemetry- } \\
(\mathbf{n = 1 6})\end{array}$ & p value \\
\hline Mean days on telemetry (Total) & 7.9 (SD: 8.6$)$ & 9.5 (SD 18.7) & 0.7396 \\
\hline Mean days to event (Shockable) & 6 (SD: 5.5) & 30 (SD 31.3) & 0.2232 \\
\hline $\begin{array}{l}\text { Mean days to event } \\
\text { (Non-Shockable) }\end{array}$ & 8.55 (SD: 9.3$)$ & 2.7 (SD 1.9) & $<0.00001$ \\
\hline
\end{tabular}

CCM: Continuous Cardiac Monitoring 


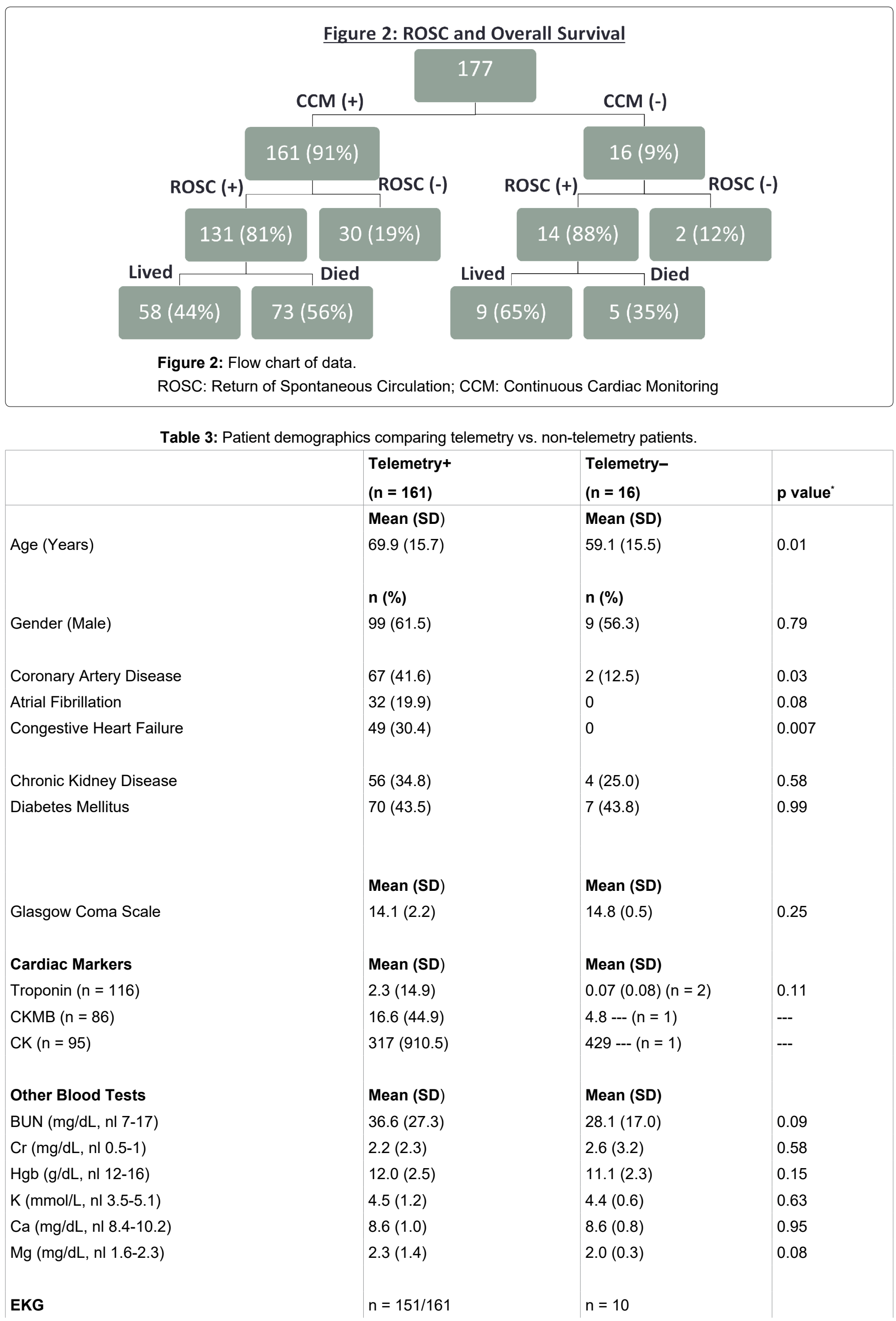




\begin{tabular}{|c|c|c|c|}
\hline & $\begin{array}{l}\text { Telemetry+ } \\
(\mathrm{n}=161)\end{array}$ & $\begin{array}{l}\text { Telemetry- } \\
(\mathrm{n}=16)\end{array}$ & p value ${ }^{*}$ \\
\hline & Mean (SD) & Mean (SD) & \\
\hline Heart Rate (beats per minute) & $90.0(26.5)$ & $82.4(19.0)$ & 0.25 \\
\hline \multirow[t]{2}{*}{ QTc (milliseconds) } & $471(44)$ & $452(38)$ & 0.18 \\
\hline & n (\%) & n (\%) & \\
\hline T-wave abnormality & $38(24.8)$ & $1(10.0)$ & 0.45 \\
\hline Sinus Rhythm & $65(43.0)$ & $7(70.0)$ & 0.11 \\
\hline Sinus Tachycardia & $33(21.9)$ & $1(10.0)$ & 0.69 \\
\hline Sinus Bradycardia & $8(5.3)$ & $2(20.0)$ & 0.12 \\
\hline Atrial Fibrillation & $29(19.2)$ & 0 & 0.24 \\
\hline Ventricular Paced & $10(6.6)$ & 0 & 0.21 \\
\hline Junctional Rhythm & $2(1.3)$ & 0 & 0.99 \\
\hline Atrioventricular Paced & $1(0.7)$ & 0 & 0.99 \\
\hline Ectopic Atrial Rhythm & $1(0.7)$ & 0 & 0.99 \\
\hline \multirow[t]{2}{*}{ Supraventricular Tachycardia } & $2(1.3)$ & 0 & 0.99 \\
\hline & Mean (SD) & Mean (SD) & \\
\hline WBC (K/mcL, nl 3.8-11) & $11.6(10.8)$ & $12.1(9.9)$ & 0.76 \\
\hline Temperature (Celsius) & $36.6(2.2)$ & $36.8(0.3)$ & 0.44 \\
\hline Heart Rate & $88.7(15.0)$ & $83.2(21.1)$ & 0.31 \\
\hline Respiratory Rate & $19.6(3.4)$ & $17.8(2.1)$ & 0.04 \\
\hline \multirow[t]{2}{*}{$\mathrm{SpO}_{2} \%$} & $96.5(3.8)$ & $97.4(2.2)$ & 0.18 \\
\hline & n (\%) & n (\%) & \\
\hline Room Air & $112(69.6)$ & $11(68.8)$ & $0.13^{* *}$ \\
\hline Nasal Cannula & $17(10.6)$ & $4(25.0)$ & \\
\hline \multirow[t]{2}{*}{ Other Oxygen delivery device } & $32(19.9)$ & $1(6.3)$ & \\
\hline & Mean (SD) & Mean (SD) & \\
\hline Systolic Blood Pressure mmHg & $124(26)$ & $120(21)$ & 0.52 \\
\hline Diastolic Blood Pressure mmHg & $70(15)$ & $69(12)$ & 0.74 \\
\hline
\end{tabular}

Laboratory, EKG, and vital sign variables recorded at earliest value from admission. Glasgow Coma Scale values recorded post return of spontaneous circulation (ROSC).

CK-MB: Creatine Kinase Muscle/Brain; CK: Creatine Kinase; BUN: Blood Urea Nitrogen; Cr: Creatinine; Hgb: Hemoglobin; K: Potassium; Ca: Calcium; Mg: Magnesium; EKG: Electrocardiogram; QTc: Corrected QT interval; WBC: White Blood Cell; SpO $\%$ : Pulse Oximetry

"p-values calculated from two-sample t-test for continuous variables and Fisher's exact test for categorical variables.

"p by Fisher's exact test for overall oxygen delivery.

while not monitored and the high percentage (91\%) of patients who triggered a code blue event that were on telemetry suggests that the American Heart Association guidelines for appropriate initiation of telemetry encompass the majority of contemporary patients at risk. Patients suffering from non-shockable causes of a code blue event while monitored on telemetry had no significant difference in survival to hospital discharge compared to those with shockable causes (41 vs. $35 \%$, p $=0.63$ ). . This paradox of successful telemetry monitoring without mortality benefit further supports the notion of the changing etiology of inpatient codes to nonshockable etiologies, an unintentional referral bias of the more ill or elderly patient, and queries whether the traditional benefit of telemetry monitoring exists even when applied appropriately.

The application of simple telemetry monitoring is often perceived by hospitalists as another "vital sign" that should be provided to those patients in whom the physician anticipates an uncertain clinical course in non-critical hospital beds. However, telemetry 
monitoring requires significant nursing resources, can create alarm fatigue and unnecessary patient interruptions, and has traditionally been reserved for those patients at significant risk who could benefit from immediate arrhythmia detection or treatment. A previous study demonstrated that efforts to improve the appropriateness of telemetry implementation was related to reduced costs while maintaining patient safety $[8,9]$. Our study demonstrated evidence of a significant proportion of patients having Class II indications, reflective of physician bias in referral of those admitted with noncardiac complaints.

Conflicting data exists regarding survival of telemetry monitored in patients suffering from a code blue event. Previously, Cleverly, et al. demonstrated $66 \%$ survival in telemetry monitored patients, versus $34 \%$ survival in un-monitored patients. Later, a conflicting study was published by Mohammad, et al. who demonstrated no survival benefit in either monitored or un-monitored patients in non-critical care beds. Both studies identified a large percentage of non-shockable rhythms as the initial inciting arrhythmia. Some important differences between the two data sets may explain the conflicting data. First, the patient population described by Cleverly, et al. only had $18 \%$ of those who experienced an arrest on telemetry monitoring. In contrast, our data and the data by Mohammad, et al. demonstrated rates of $91 \%$ and $65 \%$, respectively. Second, cardiac monitoring was capable in only $4.5 \%$ of non-critical beds in the data published by Cleverly, et al. Also, our data reveals that longer hospital stays, elevated troponin levels and age were negatively correlated with survival to hospital discharge (Table 4). We suspect the conflicting results are due to heterogenous implementation of telemetry, referral biases, and hospital telemetry logistics and availability.

As a limited bed resource, the initiation and duration of telemetry monitoring beyond 48 hours is often queried by those cost-conscious in hospital medicine. In our observations, the average length of the telemetry was 7.9 days (SD \pm 8.6 ) before a code blue event occurred, suggesting that physician "sense" of a deteriorating patient may have resulted in prolonged monitoring. Of patients with non-arrhythmic causes of a code blue activation, 33\% (44/135) initially experienced a respiratory event. The high percentage suggests that respiratory monitoring with oxygen saturation and other telemetry parameters may help with early identification of patients experiencing a respiratory cause of their code blue activation. One study evaluating changes in telemetry patterns prior to in-hospital cardiac arrest demonstrated that decreasing heart rate by a mean of three beats per minute and QRS segment morphology changes one hour prior to cardiac arrest were statistically different compared to previous hours [10]. However, few data exist evaluating the relationship among routine respiratory monitoring, identifying those at highest risk, and clinical outcomes [11]. Alternatively, increased clinical visits by dedicated inpatient nursing teams, the emerging critical care outreach model, may also serve to identify those patients on telemetry who are deteriorating in the critical week prior to their code blue activation [12].

Our study had a few important limitations. First, the sample size of our study was small and designed as a single center retrospective review attempting to observe current hospital trends in telemetry use in a typical community hospital. Patients on telemetry were older, which may reflect physician referral bias and also a lesser response to resuscitative efforts. Therefore, it is possible telemetry offered a slight benefit that was countered by the older age of the patient. The lack of non-monitored patients who suffered a code blue activation with a shockable rhythm, could represent degeneration of a initial shockable rhythm into asystole because of a delay in recognition. Implementation of telemetry, despite the AHA guidelines may differ among different institutions based on local practice differences. Lastly, we included respiratory code blues in the non-shockable causes of cardiac arrest, which are not specifically addressed in the ACLS guidelines until their underlying heart rhythm converts to ventricular tachycardia or fibrillation, pulseless electrical activity or asystole. Accordingly, our telemetry data cannot specifically address whether a shockable cause was initially incited by a respiratory or metabolic event.

\section{Conclusion}

In summary, our retrospective analysis has demonstrated that a large percentage of inpatient code

Table 4: Characteristics associated with survival.

\begin{tabular}{|l|l|l|l|l|}
\hline & \multicolumn{2}{|c|}{ Unadjusted } & \multicolumn{2}{c|}{ Adjusted $^{*}$} \\
\hline Characteristic & Odds Ratio (95\% Confidence Interval) & $p$ & Odds Ratio (95\% Confidence Interval) & P \\
\hline Age (10 yr. intervals) & $0.7(0.6,0.8)$ & 0.0004 & $0.7(0.5,0.8)$ & $<0.001$ \\
\hline AHA Class 3 & $3.0(1.0,8.6)$ & 0.04 & $1.5(0.5,5.0)$ & 0.49 \\
\hline $\begin{array}{l}\text { Days from admission to } \\
\text { code (log) }\end{array}$ & $0.7(0.5,1.0)$ & 0.02 & $0.6(0.4,0.8)$ & 0.002 \\
\hline $\begin{array}{l}\text { Troponin level }>0.04 \text { on } \\
\text { admission }\end{array}$ & $0.3(0.2,0.7)$ & 0.002 & $0.4(0.2,0.8)$ & 0.01 \\
\hline
\end{tabular}

*Logistic regression model including all 4 listed variables. (Odds ratios $<1.0$ indicate negative association with survival). 
blues were appropriately monitored on telemetry, but were due to non-shockable causes and occurred late in hospitalization. We demonstrate no significant survival difference in those patients who were monitored on telemetry suffering from either a shockable versus nonshockable cause of their code blue. Further studies to identify earlier clinical deterioration in patients who suffer from non-shockable etiologies and novel, less expensive approaches to monitoring such as could potentially be appropriate.

\section{Funding Sources}

None.

\section{Conflict of Interest}

No significant interests or competing interests to disclose.

\section{References}

1. Sandau KE, Funk M, Auerbach A, Barsness GW, Blum K, et al. (2017) Update to practice standards for electrocardiographic monitoring in hospital settings: A scientific statement from the American Heart Association. Circulation 136: e273-e344.

2. Najafi N (2014) A call for evidence-based telemetry monitoring: The beep goes on. JAMA Intern Med 174: 1855-1856.

3. Zorzi A, Peruzza F, Stella F, Del Monte A, Migliore F, et al. (2016) Life-threatening ventricular tachyarrhythmias in the cardiology department: Implications for appropriate prescription of telemetry monitoring. Resuscitation 101: 6-11.
4. Cleverley K, Mousavi N, Stronger L, Ann-Bordun K, Hall $\mathrm{L}$, et al. (2013) The impact of telemetry on survival of in-hospital cardiac arrests in non-critical care patients. Resuscitation 84: 878-882.

5. Mohammad R, Shah S, Donath E, Hartmann N, Rasmussen A, et al. (2015) Non-critical care telemetry and in-hospital cardiac arrest outcomes. J Electrocardiol 48: 426-429.

6. Girotra S, Nallamothu BK, Spertus JA, Li Y, Kumholz HM, et al. (2012) Trends in survival after in-hospital cardiac arrest. N Engl J Med 367: 1912-1920.

7. Falun $\mathrm{N}$, Nordrehaug JE, Hoff $\mathrm{P}$, Langorgen J, Moons $\mathrm{P}$, et al. (2013) Evaluation of the appropriateness and outcome of in-hospital telemetry monitoring. Am J Cardiol 112: 12191223.

8. Svec D, Ahuja N, Evans KH, Hom J, Garg T, et al. (2015) Hospitalist intervention for appropriate use of telemetry reduces length of stay and cost. J Hosp Med 10: 627-632.

9. Benjamin EM, Klugman RA, Luckmann R, Faichild DG, Abookire SA (2013) Impact of cardiac telemetry on patient safety and cost. Am J Manag Care 19: e225-e232.

10. Attin M, Feld G, Lemus $H$, Najarian K, Shandilya $S$, et al. (2015) Electrocardiogram characteristics prior to in-hospital cardiac arrest. J Clin Monit Comput 29: 385-392.

11. Andersen LW, Holmberg MJ, Berg KM, Donnino MW, Granfeldt A (2019) In-hospital cardiac arrest: A review. JAMA 321: 1200-1210.

12. Ball C, Kirkby M, Williams S (2003) Effect of the critical care outreach team on patient survival to discharge from hospital and readmission to critical care: Non-randomised population based study. BMJ 327: 1014 